\title{
Lógicas emergentes de acción colectiva y prácticas colaborativas de investigación. Apuntes para una Antropología junto y con los movimientos sociales
}

\author{
Emerging logic in collective action and collaborative research practices: notes for an Anthropology with and \\ for social movements
}

\author{
Alberto Arribas Lozano \\ Candidato doctoral del Departamento de Antropología Social. Universidad de Granada. España \\ aarribas@ugr.es
}

\begin{abstract}
RESUMEN
Este artículo es una reflexión sobre la relación entre la Antropología y el estudio de los movimientos sociales. En primer lugar, presento la aparición de lógicas y prácticas emergentes en el campo de la acción colectiva, nuevos protagonismos sociales cuyas características difieren de la imagen clásica de un actor político organizado. Después paso a explicar las razones por las que considero que la Antropología está mejor preparada para captar este tipo de procesos que los enfoques hoy dominantes en el estudio de los movimientos sociales, ya que la el trabajo de campo etnográfico permite aprehender estas dinámicas emergentes según se están construyendo y desplegando. Y finalmente planteo las discusiones epistemológicas y metodológicas que me llevan a defender la etnografía colaborativa -investigar junto y con los movimientos sociales y no sobre los movimientos socialescomo la aproximación que, a la vez que nos sirve para producir conocimiento en torno a estas experiencias emergentes, permite que nuestros proyectos resulten relevantes para los sujetos con quienes trabajamos.
\end{abstract}

\section{ABSTRACT}

This work analyses the relationship between Anthropology and the study of social movements. First I introduce the emergence of new types of logic and practices in the field of collective action, which sharply differ from our previous images of organized political actors. Then I explain why Anthropology is particularly suitable to grasp these types of processes, much more so that the dominant approaches in Social Movement Studies, since ethnographic fieldwork enables us to apprehend emerging dynamics as they are being built and deployed. I conclude the article by bringing collaborative anthropology into the discussion, emphasizing the epistemological and methodological features that make this proposal appropriate for implementing research projects simultaneously relevant for the academia as well as for the subjects and social movements that we are working with.

PALABRAS CLAVE

antropología colaborativa | movimientos sociales | compañeros epistémicos | reflexividad | acción colectiva emergente KEYWORDS

collaborative Anthropology | social movements | epistemic partners | reflexivity | emerging collective action

\section{Introducción}

Estamos asistiendo en los últimos años a transformaciones profundas en las lógicas y prácticas de la acción colectiva. Emergen nuevos protagonismos sociales a partir de la diversificación y redefinición de los sujetos, espacios y demandas políticas (Norris 2002), así como de las formas organizativas, los discursos y los repertorios de acción que orientan y expresan la (re)invención de unos movimientos sociales que no se ajustan ya -si es que alguna vez lo hicieron- al modelo clásico de entidades unitarias y homogéneas claramente delimitadas. Hoy nos encontramos más bien ante estructuras reticulares complejas y áltamente heterogéneas, cuyos contornos se muestran imprecisos, desordenados y cambiantes; entramados y circuitos de solidaridad que difieren profundamente de la imagen de un actor político organizado (Diani 2003, Mendiola 2003). No hablaríamos, por lo tanto, de totalidades cerradas sino de una topología compleja de redes enredadas, constelaciones dinámicas de prácticas, afectos, herramientas y sentidos compartidos, cuyo carácter abierto -poroso, indefinido, discontinuo- no se entiende como un obstáculo para la acción colectiva, sino como uno de los elementos constitutivos de las formas de pensar, imaginar y hacer otra política hoy. 
Este carácter difuso no remite únicamente a la forma o la estructura de estas experiencias novedosas, también sus criterios de pertenencia están poco codificados; su propuesta no pasa ya por (re)crear identidades colectivas fuertes ni por proponer posicionamientos ideológicos muy marcados, al considerase que en lugar de facilitar la construcción de movimiento estos acaban convirtiéndose en un obstáculo para la acción colectiva (1), sino que se busca (aprender a) intervenir políticamente -producir ideas y vínculo social, trabajar en conexión con otros y otras- sin que para ello haya que pensar igual: experimentar la cooperación entre diferentes sin negar las diferencias, hacer proliferar las prácticas organizativas sin centralizarlas, sentirse y saberse parte de procesos comunes sin construir para ello identificaciones rígidas. Estaríamos así ante lógicas de afinidad, "formas de coordinación de singularidades que constituyen sumas que no totalizan sus propios elementos" (Lazzarato 2006: 65). Y desde estas premisas se está abriendo paso un modelo de acción colectiva que podríamos denominar post-identitaria y post-ideológica, siempre que entendamos que estas categorías no remiten a una propuesta post-política o antipolítica, sino al deseo compartido de producir colectivamente otra política, donde la diversidad no es tomada como problema sino como punto de partida, horizonte y desafío. De este modo, en el marco de la sociedad red la gramática de las luchas (el conflicto social) se despliega cada vez más en términos de complejidad, afinidad, conexión y cooperación entre singularidades, una situación que nos lleva a tener que problematizar la idea de un 'nosotros' claramente diferenciado como precondición necesaria para la acción (Mcdonald 2002, Chesters y Welsh 2006, Flesher 2010).

Se trata de un panorama novedoso que aún estamos aprendiendo a nombrar; redes difusas que conforman una ecología de interrelaciones complejas y cambiantes (Holmes 2006: 92), y que se componen desde la construcción de vínculos -políticos y afectivos- que deben producirse y reproducirse, cuidarse y tejerse en la materialidad y en la inmanencia de las prácticas y luchas cotidianas. Estaríamos aquí ante un desplazamiento fundamental. No hablamos ya de una arquitectura de los grandes diseños o relatos políticos que se despliegan en planes ideales (ingeniería social, saberes expertos, teorías de vanguardia); sino que estamos, en su lugar, ante una concepción de la política como artesanía (Osterweil y Chesters 2007), una experimentación abierta y siempre en construcción donde el encuentro y las prácticas no derivan ya, como he dicho antes, de los corpus ideológicos o las identidades fuertes, sino de articularse en torno a problemas y malestares sociales compartidos y concretos que se expresan y se viven en ese ámbito cotidiano. Es importante insistir en esta idea: si lo cotidiano es el plano espacio/temporal central para la reproducción de las relaciones sociales capitalistas y para la vivencia de sus consecuencias más concretas -hoy, por ejemplo, la experiencia encarnada de la precariedad- parece lógico entonces que se convierta también en el campo privilegiado de las luchas, las resistencias y los proyectos colectivos de (re)invención de otras maneras de vivir y convivir. Estamos así ante una política de la vida cotidiana, un marco que debería ser también, por lo tanto, el plano en el que despleguemos nuestras investigaciones.

Un buen ejemplo de estas dinámicas emergentes que vengo señalando son las manifestaciones celebradas el 15 de mayo de 2011, y los sucesos de las semanas y meses posteriores (Castells 2012, Taibo 2011 y 2012, Cruells e lbarra 2013, Calle 2013) (2), que denominaré como el acontecimiento/movimiento 15M. En esta línea, Fernández-Savater nos proponía pensar dicha experiencia en clave de "identidades no identitarias" (Fernández-Savater 2012: 678) y como "movimientos sociales que no son movimientos sociales" (Fernández-Savater 2012: 669). Reflexionando en clave de continuidades y discontinuidades, podríamos decir que estos elementos -estas formas novedosas de imaginar y reinventar la política desde abajo- ya estaban presentes, ya estaban siendo pensados y puestos a trabajar en diversos proyectos que venían experimentando en los últimos años en torno a las formas de hacer y a la redefinición de los dispositivos y las prácticas, pero se trataba de procesos aún muy minoritarios (3); y será en el contexto del acontecimiento/movimiento $15 \mathrm{M}$ cuando se expresen de manera multitudinaria. El $15 \mathrm{M}$ materializaba muchas de esas intuiciones políticas, pero lo hacía a una escala, un ritmo y una velocidad mucho más amplias; lo que había sido minoritario, devenía en las calles y plazas el sentido común compartido por muchos y muchas: la auto-organización, la producción de una política que no pase por la ideología o la identidad, la problematización de los malestares cotidianos, los espacios de encuentro entre diferentes, etcétera. De hecho estas ideas atravesaban el manifiesto de convocatoria de la movilización del 15 de mayo de 2011, que alejándose de los discursos militantes al uso, decía:

"Somos personas normales y corrientes. Somos como tú: gente que se levanta por las mañanas para estudiar, para trabajar o para buscar trabajo, gente que tiene familia y amigos. Gente que trabaja duro todos los días para vivir y dar un futuro mejor a los que nos rodean. 
Unos nos consideramos más progresistas, otros más conservadores. Unos creyentes, otros no. Unos tenemos ideologías bien definidas, otros nos consideramos apolíticos. Pero todos estamos preocupados e indignados por el panorama político, económico y social que vemos a nuestro alrededor. Por la corrupción de los políticos, empresarios, banqueros. Por la indefensión del ciudadano de a pie.

Esta situación nos hace daño a todos diariamente. Pero si todos nos unimos, podemos cambiarla. Es hora de ponerse en movimiento, hora de construir entre todos una sociedad mejor" (Democracia Real Ya! 2011) (4).

Y fue este tipo de planteamiento el que produjo la interpelación masiva que posibilitó la apertura de un nuevo escenario en aquellas semanas, una interrupción inesperada de la normalidad que abría nuevas posibilidades para el pensamiento y la acción, y que situaba en el centro del debate preguntas y opciones que no estaban dadas de antemano. En ese sentido, es importante destacar que el acontecimiento/movimiento $15 \mathrm{M}$ sucedió por afuera de los movimientos sociales organizados y de los circuitos activistas tradicionales, pasó por otro(s) lado(s), hablándonos así de esas formas emergentes de la acción colectiva que se constituyen como un espacio de cualquiera (Fernández-Savater 2012: 678). Como hemos visto, desde su inicio la convocatoria para la movilización remitía con fuerza a un imaginario y un lenguaje muy inclusivos, y presuponía así la posibilidad de una comunidad abierta; los lemas que circulaban eran transversales, llamaban a muchos y muchas e invitaban a producir colectivamente esa otra política. Potencialmente cualquiera podía sentirse interpelado por una protesta contra el bajo perfil de la democracia española: una ley electoral discutible, altos niveles de corrupción, poca transparencia, ausencia de canales de participación, etc.; y también cualquiera podía sentir que había que dar alguna respuesta a la creciente precarización de nuestras vidas, y en ese sentido incidía en esa idea de dar prioridad a los problemas concretos y cotidianos, sentidos en primera persona, por encima de los planteamientos de corte más ideológico e identitario. Y esto es algo que hemos podido ver también en las diversas mareas contra los recortes presupuestarios y de derechos en educación, sanidad, etc., así como en la experiencia de la Plataforma de Afectados/as por la Hipoteca, que en la actualidad es tal vez el proyecto más sólido y con mayor capacidad de incidencia; son dinámicas de intervención más específicas que el acontecimiento/movimiento $15 \mathrm{M}$ pero que funcionaban desde esas mismas lógicas emergentes (la política como artesanía). $\mathrm{Y}$ nuestras investigaciones, nuestras preguntas y maneras de analizar no pueden permanecer ajenas a estos cambios.

\section{¿Cómo mirar? Hacia una antropología de los movimientos sociales}

En este marco de transformación de las lógicas y las prácticas de la acción colectiva es importante plantearnos algunos interrogantes. No es fácil pensar ni comprender estos procesos emergentes a partir de las categorías e imágenes que hasta ahora nos permitían reconocer a los movimientos sociales, ya que "la constitución actual de lo común no pasa por ninguna de las figuras de lo común que están instaladas en nuestro imaginario" (Pelbart 2009: 12). ¿Están sucediendo cosas que no vemos -no podemos ver- con los instrumentos, categorías y preguntas de que disponemos?, ¿nos sirven los mapas que tenemos para aproximarnos a fenómenos que implican una ruptura -una asimetría- con lo ya conocido? Si esa otra política de los movimientos sociales no está vinculada a una ideología o a una identidad compartidas a priori, entonces ¿cómo se construyen, cómo se tejen y se sostienen los vínculos que hacen posible la acción colectiva?, ¿cómo toman cuerpo esa otra política y esos otros protagonismos sociales que actúan desde y hacia la heterogeneidad? ¿Qué instrumentos, qué herramientas de análisis necesitamos para dar cuenta de procesos abiertos, de lógicas en movimiento, de configuraciones cambiantes?, y ¿cómo afecta esto al trabajo de campo y a las lógicas de representación?, ¿cómo organizar las imágenes, los discursos, las metáforas que expresan la complejidad de estos fenómenos?, ¿cómo aprehender -sin cosificarlas- sus características, sus innovaciones y continuidades?

Mi propuesta en este artículo es que los estudios hegemónicos sobre movimientos sociales presentan cierta rigidez a la hora de captar estos procesos que están en construcción. La aparición de lo que Melucci definía críticamente como un "mercado académico mundial en el campo de la investigación sobre movimientos sociales" (Melucci 1989: 195) (5) provocó que la producción de conocimiento en este área gire ahora, mayoritariamente, en torno a los problemas, discusiones y preguntas internas de la propia subdisciplina, 
imponiendo así las categorías analíticas del propio campo sobre los sujetos investigados, en una lógica autorreferencial que ha provocado que los estudiossobremovimientos sociales hayan perdido en gran medida su capacidad de diálogo con los movimientos sociales (Cox y Flesher 2009, Croteau et al. 2005).

Esto no siempre fue así. En su origen, el proceso de elaboración y acumulación teórica y empírica que posibilitó el surgimiento del estudio de los movimientos sociales como subdisciplina se produjo -en el ámbito norteamericano, que devendría eventualmente hegemónico- en el contexto marcado por las luchas sociales de las décadas de 1960 y 1970, el movimiento por los derechos civiles, feminista, estudiantil, o el movimiento anti-imperialista y contra la guerra de Vietnam, cuando académicos y académicas que tenían fuertes vínculos con estos procesos, o que eran directamente activistas, desafiaron las interpretaciones que en ese momento eran dominantes en el análisis de la acción colectiva. Ésta se entendía principalmente como la expresión de conductas irracionales y anómicas, lo que despolitizaba por completo su carácter y su sentido, y fue gracias a este gran impulso colectivo que pudo comenzar a analizarse desde una mirada que subrayaba el papel de los movimientos como agentes centrales en la transformación social y política de nuestras sociedades. Por ejemplo Doug McAdam, una de las figuras clave en el desarrollo de estos estudios, contaba así su propia experiencia:

"Mi primera exposición al estudio académico de los movimientos sociales tuvo lugar en 1971 cuando, para mi sorpresa, el profesor de la clase de Psicopatología dedicó varias semanas a la discusión de este tema. Digo 'sorpresa' porque, como participante activo en el movimiento anti-guerra, ciertamente fue una noticia saber que mi implicación en la lucha era debida a una mezcla de patología personal y desorganización social. Pero esos fueron, reflejando las teorías dominantes en aquel momento, los dos factores que se subrayaron en el curso." (McAdam 2003: 281) (6).

El cambio que se produjo en ese contexto de efervescencia política y social fue extraordinario, e hizo posible abrir nuevos horizontes y programas de investigación. Sin embargo, mi sensación es que más allá de lo logrado -que es mucho- en este proceso de institucionalización y profesionalización los estudios sobre movimientos sociales han ido perdiendo progresivamente esa relación intensa entre academia y activismo que sí estaba presente en su origen, generándose así un distanciamiento creciente que ha bloqueado la posibilidad de elaborar preguntas compartidas (Bevington y Dixon 2005, Meyer 2005, Goodwin y Hetland 2009). Así por ejemplo, Richard Flacks afirmaba que

"Era posible imaginar, si estabas implicado en el campo de los estudios sobre movimientos sociales, que tus clases, tu asesoramiento y tu participación directa, así como tus esfuerzos de investigación, podían tener alguna relevancia para las prácticas y los puntos de vista de los activistas políticos. En algún punto a lo largo del camino, sin embargo, esa promesa de relevancia retrocedió (se esfumó) y una definición de objetivos mucho más 'profesional' y 'disciplinaria' pasó a primer plano" (Flacks 2004: 136) (7).

De este modo, dicha subdisciplina se fue transformando en una ciencia sedentaria, auto-referencial, que no se interesa ya por acompañar aquello que de movimiento tienen los movimientos, y que convierte en objetos a los sujetos con quienes trabaja, a los que trata como materia prima para su propia reproducción (8). Y en ese sentido, la inercia de su aparataje conceptual y teórico -que circula y se reproduce en las revistas y conferencias académicas especializadas- es en cierto modo un obstáculo para percibir y analizar dinámicas que están emergiendo.

¿Qué puede aportar en este contexto la antropología? Cartografiar lo emergente implica focalizar la mirada en los procesos según se producen y se despliegan, y para eso hay que situarse al interior de esas redes de relaciones, escuchar los discursos, conocer las prácticas, percibir la textura, el ritmo y las tonalidades de esas tramas de sentido que se construyen colectivamente. Y eso se logra a través de la inserción en el tiempo y el espacio de lo cotidiano que caracteriza al trabajo de campo etnográfico, donde como investigadores o investigadoras observamos qué sucede, escuchamos, preguntamos, sentimos, dialogamos y participamos en mayor o menor medida (9). La combinación metódica entre lo diacrónico y lo sincrónico, la atención privilegiada al proceso y a lo relacional, a lo micro-político, a esa (re)producción y transformación de los vínculos y los sentidos compartidos, es lo que va a hacer posible acercarnos a la multidimensionalidad inherente a estas experiencias reticulares y difusas; y ahí la etnografía es clave porque nos invita a mirar de manera diferente, prestando mayor atención a la contingencia, la diversidad, el dinamismo y la complejidad de los factores que se (re)combinan de maneras inesperadas. De este modo, 
por su carácter de disciplina indisciplinada (Comaroff 2010), y por el enorme potencial que tienen sus técnicas de producción de conocimiento para abrir nuevos horizontes de investigación y percibir lo que está por llegar, lo que está naciendo, la antropología puede jugar un papel fundamental a la hora de detectar y analizar este tipo de procesos emergentes (Hannerz 2010).

Por otro lado, he señalado cómo estas redes se conforman como entramados heterogéneos, abiertos, compuestos por una multiplicidad de niveles y cuya topología no viene dada de antemano. En este sentido, Latour afirmaba que ante este tipo de procesos donde los límites no están claramente definidos y donde las dimensiones a considerar fluctúan, la tarea del investigador o investigadora no sería imponer a priori algún tipo de orden, limitando la forma, el tamaño o el carácter de las conexiones, sino "seguir a los propios actores" (Latour 2005: 12), es decir, que sean los propios sujetos -los y las integrantes de los movimientos sociales en este caso- quienes tracen la particular geografía variable de su campo. Y aquí de nuevo la etnografía se nos muestra como una herramienta extremadamente útil, ya que no pretende sobre-codificar los discursos de los sujetos desde las categorías disciplinares, sino abrir el espacio necesario para que sean esos sujetos quienes definan el sentido (los sentidos) de sus prácticas, y propongan, desplieguen y ordenen sus propios conceptos, análisis y mapas de relaciones (Auyero y Joseph 2007, Hammersley y Atkinson 1994, Schatz 2009).

Además, y como último punto, la antropología aporta también su preocupación particular por los elementos culturales, y esto es especialmente importante si entendemos que uno de los planos fundamentales de acción de los movimientos sociales es (re)nombrar el mundo de maneras alternativas, creando y resignificando códigos e imaginarios -relatos, ideas, mitos, figuras- y ampliando así el campo de lo posible y lo pensable. Las redes subterráneas de movimientos sociales funcionan como espacios de experiencia y experimentación en los que, "se plantean nuevos problemas y preguntas, y en los que se inventan y ensayan nuevas respuestas" (Melucci 1989: 208) (10), y operan por lo tanto como "laboratorios de innovación cultural" (McAdam 1994: 55), activando un trabajo cotidiano en torno a las representaciones sociales, a la producción simbólica y a los procesos y dispositivos de subjetivación, que aparecen hoy como un ámbito clave de intervención para la acción colectiva (Escobar y Osterweil 2009).

Por todo esto, la antropología sería un enfoque privilegiado para aproximarnos a este tipo de dinámicas emergentes. Sin embargo, y a pesar de las características que acabo de mencionar, la Antropología -en comparación con la Sociología, la Historiografía o la Ciencia Política- ha tenido hasta ahora un papel bastante limitado en este campo de análisis. Hace más de dos décadas, Arturo Escobar lamentaba en un texto ya clásico la invisibilidad de los movimientos sociales en la antropología, afirmando que la investigación de algo tan heterogéneo y complejo como los movimientos sociales contemporáneos suponía de hecho un desafío que podía ayudar a profundizar la autocrítica de la disciplina, teniendo implicaciones importantes para el trabajo de campo y para la dimensión política de la escritura etnográfica, es decir, para quién escribimos y cómo; y planteando así cuestionamientos epistemológicos y metodológicos que podían ser muy fructíferos en términos de cruces innovadores entre teoría y práctica, conocimiento y acción (Escobar 1992: 419). Y más adelante, Javier Auyero (2005: 122) insistía en esta ausencia, destacando que hay mucha reconstrucción pos facto de la protesta pero que la producción de etnografía in situ sigue siendo escasa.

Esta invisibilidad, que por supuesto nunca fue completa, parecería no obstante estar cambiando a lo largo de la última década, y con mayor intensidad aún al calor de los movimientos sociales que han surgido en los últimos años. Así, por citar algunos ejemplos, puede destacarse la creación de una red de investigadores e investigadoras en antropología de los movimientos sociales dentro de la European Association of Social Anthropologists (11); la presencia creciente de esta temática en congresos de antropología, y la publicación de manuales específicos como el caso de Nash (2005), Grimberg, Macedo y Manzano (2011), o Juris y Khasnabish (2013); la edición del monográfico "Etnografías de la indignación" coordinado en 2013 por Fernández de Rota y Diz en la Revista de Antropología Experimental(12); los múltiples materiales de la carpeta "Occupy, Anthropology, and the 2011 Global Uprisings", publicada por Cultural Anthropology en 2012 (13); los trabajos de Estalella y Corsín en torno al 15M en el proyecto Prototyping (14); así como algunos de los artículos editados por la revista Collaborative Anthropologies(15), o los presentados por el Berkeley Journal of Sociology en su foro "Understanding the Occupy Movement: Perspectives from the Social Sciences" (16). Creo que este breve recuento nos da una idea de cómo los investigadores e investigadoras parecen estar buscándose desde la necesidad y el deseo de debatir sobre intereses y problemáticas compartidas. 


\section{Un paso más allá. La dimensión colaborativa: de la relación sujeto/objeto de investigación a un diálogo de reflexividades}

Sin embargo, y junto a estos elementos que vengo subrayando, creo que es importante dar un paso más allá en el análisis de las formas emergentes de la acción colectiva (17). Si no hay un trabajo epistemológico fuerte que oriente explícitamente nuestros diseños metodológicos en otra dirección, es probable que acabemos practicando por defecto etnografía sobre los movimientos sociales, y en ese caso estaremos dejando sin problematizar -y por lo tanto, reproduciremos- la asimetría y la subordinación que componen y atraviesan la relación sujeto/objeto de investigación (18). Para evitar que esto suceda es clave situar las preguntas ¿para qué? y ¿para quién? en el centro de nuestros proyectos; y en ese sentido Escobar (2008) enfatizaba la importancia de desarrollar nuestros trabajos pensando junto y con los movimientos sociales, tomando como punto de partida sus localizaciones epistémicas y políticas, y no únicamente los intereses académicos o disciplinarios. Es central además reconocer el papel de los movimientos sociales como productores de conocimiento, en un contexto en el que para muchas experiencias la producción y sistematización de saberes -situados, encarnados, creados colectivamente, surgidos en y desde las prácticas- no se piensan como un complemento o como un momento separado de la política, no son propuestas que tengan sentido aisladas: el pensamiento colectivo, la investigación militante, la experimentación política y la producción de movimiento son entendidos y vividos como hilos de un mismo tejido (Casas-Cortés, Osterweil y Powell 2008, Santucho 2012).

Ese gesto que nos propone Escobar permitiría articular investigaciones que, además de para la academia, fueran útiles para los sujetos con quienes trabajamos. Esta afirmación no esconde ningún proyecto normativo: la relevancia no puede definirse por fuera de cada situación específica, y debe ser negociada y definida colectivamente por los diferentes actores implicados; pero sería deseable que cada investigador o investigadora tuviera presente -sin dogmatismos, pero con honestidad- las cuestiones de para qué y para quién sirven los proyectos que desarrollamos. Y me estoy refiriendo aquí a la relevancia de la investigación como proceso, y no únicamente como resultado, que es como se piensa habitualmente: un textoherramienta (o de un conjunto de ellos) que da a conocer una experiencia, que visibiliza prácticas, y genera o alimenta debates relevantes para los y las activistas. $Y$ todo eso es necesario, pero lo que me interesa pensar -como pregunta fuerte- es cómo lograr que la investigación sea útil como proceso, y no únicamente como producto final, un gesto que nos obliga a discutir, negociar y articular intereses y definiciones que sean -al menos parcialmente- compartidos en relación al diseño y al desarrollo de la investigación. En mi opinión hacer posible este modelo de relevancia, darle cuerpo, pasa necesariamente por afirmar la reflexividad de los grupos con quienes trabajamos, e intentar que nuestras investigaciones se conecten y se recombinen de un modo creativo y productivo con sus propias conversaciones. No se trataría de "enseñar" nada al movimiento desde una posición de "experto", sino que, como señalaba Juris, "el conocimiento etnográfico producido de manera colaborativa busca facilitar procesos de (auto)reflexión activista que ya están en marcha en relación a los objetivos, las tácticas, las estrategias o las formas organizativas de un movimiento" (Juris 2007: 165) (19); y en ese marco la tarea del investigador o la investigadora es acompañar -aprender acompañando y siendo acompañado/a-, abriendo un espacio de reflexividad dialógica y co-análisis.

Esto conlleva diversos desplazamientos. El primero es pasar de una relación de investigación sujeto/objeto a una relación entre sujetos en proceso, donde se toma como punto de partida la condición de productores de conocimiento de los grupos con quienes trabajamos; como señalaban Holmes y Marcus.

"Dentro de las comunidades epistémicas que buscamos explorar, nuestros sujetos son perfectamente capaces de hacer magníficas etnografías en sus propios idiomas. En los términos de sus propios discursos situados, la función descriptiva básica de la etnografía es muy probable que ya se esté ejerciendo. A modo de artefactos, libros y memorias emergen cada día desde el interior de las mismas, por así decirlo, y explican con gran agudeza crítica cómo los procesos, instituciones y organizaciones contemporáneas más complejas y estratégicas operan y tienen sus propias culturas. No son necesarios los antropólogos para añadir 'crítica', orden moral o un significado superior a dichos relatos. (...) nosotros debemos, por lo tanto, reaprender nuestro método a partir de nuestros sujetos tomados como compañeros epistémicos, desde la evaluación cuidadosa de cómo ellos se involucran intelectualmente con nuestro mundo y nuestro tiempo." (Holmes y Marcus 2008: 84) (20). 
En este sentido, y como segundo desplazamiento, no estamos ya ante la categoría clásica del informante, el nativo o la nativa que proveen de información al experto, que será quien realice el análisis complejo que ellos y ellas supuestamente no podrían hacer; sino ante un escenario poblado por actores hiper-reflexivos, compañeros/as epistémicos/as, cuyas prácticas están atravesadas por el mismo ethos experimental que las del investigador, y que ponen en juego y despliegan lo que Holmes y Marcus han denominado como para-etnografía.

"Para nosotros, la figura del para-etnógrafo cambia fundamentalmente las reglas de juego de la colaboración, así como la mediación de ideas y sensibilidades englobadas por y en el intercambio etnográfico. No nos interesa la colaboración como una 'división del trabajo' entre los investigadores o investigadoras que controlan el diseño de un proyecto, ni como la base para combinar saberes expertos académicos o como un gesto hacia la interdisciplinariedad canónica. La idea es, de nuevo, integrar completamente la agudeza analítica y las percepciones de nuestros sujetos para definir las temáticas que se ponen en juego en nuestros proyectos, así como los medios a través de los cuales vamos a explorarlas" (Holmes y Marcus 2008: 86) (21).

No estaríamos por lo tanto ante el imaginario de la investigación etnográfica como caza o captura del que hablaba Malinowski, sino ante una lógica de cooperación, pensada en clave de un caminar compartido (22). Una idea que se ha venido planteando desde diferentes escenarios: la conexión entre diversos elementos de la Investigación Acción Participativa y la antropología (Greenwood 2000), las propuestas de la sociopraxis (Villasante 2006), la etnografía doblemente reflexiva (Gunther Dietz 2011), o los aportes de la antropología feminista y las metodologías decoloniales son algunos de los enfoques y herramientas que nos permiten acercarnos a ese horizonte colaborativo que vengo señalando, y cuyas principales características voy a detallar a continuación.

En primer lugar, las propuestas colaborativas implican repensar/resignificar la práctica de colaboración -que es por definición inherente a la etnografía- para situarla deliberada y explícitamente como la columna vertebral que oriente y dé sentido al diseño de todas y cada una de las fases de la investigación: desde la formulación del proyecto hasta el trabajo de campo, el análisis y la escritura, poniendo en discusión con los sujetos con quienes trabajamos las representaciones que serán elaboradas a partir de los datos producidos (Lassiter 2005: 132). La colaboración se entiende, por lo tanto, como producción conjunta e implica idealmente un proceso continuo de diálogo, ensayo y (re)negociación de una agenda compartida y de los objetivos -no siempre coincidentes- de los diferentes actores.

En este sentido, situar las prácticas colaborativas como eje del proceso de investigación modifica profundamente el carácter del trabajo de campo, que pasa de ser un espacio/tiempo de producción o recolección de datos, anterior y separado del momento del análisis, a ser un espacio/tiempo en el que se despliegan dinámicas colectivas (talleres, cartografías, etc.) de co-teorización y co-conceptualización (Vasco 2002, Rappaport 2007 y 2008) que serán los que den lugar a la producción de conocimiento. Por eso no tiene sentido hablar, como sí hacen otras propuestas, de una fase posterior de devolución del análisis elaborado por el investigador/experto, sino que nos estamos situando en otro plano, en una lógica de la investigación como artesanía, en la que lo importante es pensar juntos y juntas: abrir espirales de "acción-reflexión-acción" en las que la reflexión colectiva sobre la práctica vaya construyendo nuevas reflexividades y posibilidades de acción más creativas. Un escenario, por lo tanto, en el que es central el cuidado del proceso, y donde nuestra tarea como investigadores e investigadoras es situarnos en clave de transducción, entendiendo que el análisis es compartido y que ésa es justamente la mayor riqueza de estos proyectos.

Es fundamental señalar, además, que al abrir espacios y tiempos de codecisión en el diseño y análisis de la investigación, la etnografía colaborativa desestabiliza la asimetría de poder (la violencia simbólica) implícita en la relación sujeto investigador/objeto investigado, estableciendo una situación en la que "para que los sujetos de la investigación tengan un grado adicional de control real, el investigador o investigadora tendrán que delegar una parte significativa de su propio control" (Hale 2008: 15) (23).

Y creo que es importante entender esta pérdida de control más como una oportunidad que como un problema; se trata de una propuesta desde la complejidad, sin garantías, experimental y que no está -no puede estar- exenta de tensiones (Leyva y Speed 2008), pero que nos permite situar con fuerza la cuestión de la relevancia (la utilidad para las personas con quienes trabajamos) en el centro del proyecto, a la vez 
que producimos análisis académicos más ricos y profundos al acceder a planos de acción a los que resultaría difícil llegar desde otras aproximaciones. Cuanto más puedan apropiarse del proyecto los sujetos con quienes trabajamos, aunque sea parcialmente, más rico va a ser el análisis compartido, y mayores nuestras opciones de observar -en su producción material y concreta- los procesos de construcción y redefinición colectiva de las categorías, los sentidos y las nociones comunes sobre las que se asienta y se despliega la acción colectiva. Entiendo que esa pérdida de control deja al investigador o investigadora en una posición ambigua, en algún lugar indefinido -entremedias- de la academia y de los movimientos, y que habitar y sostener esa situación puede activar tensiones que son difíciles de gestionar. Realizar un trabajo que resulte creíble y sólido en los dos ámbitos, el académico y el de los movimientos sociales, es tal vez la más destacada y hay un riesgo evidente en intentar mantener esta doble lealtad: acabar por no hacer bien ninguna de las dos cosas, no cumplir con las expectativas, las demandas ni los criterios de aceptación y validación de estos dos ámbitos. Pero a la vez insisto en que esa incomodidad y esa ambigüedad pueden abrirnos posibilidades inesperadas si somos capaces -junto y con los sujetos con quienes trabajamos- de aprender a habitar creativamente esa tensión e intentamos declinarla de manera productiva (Hale 2008: 23).

En este sentido, Rappaport (2008: 23) planteaba una serie de condiciones ideales para la realización de una etnografía colaborativa: en primer lugar, el compromiso de sostener un diálogo a largo plazo, lo que no siempre es posible; en segundo lugar, un grado significativo de confianza entre las partes que generalmente nace de una relación construida durante años; y como tercer factor, la presencia de un grupo de interlocutores que puedan asumir y liderar el proceso de co-teorización. Fuera del cuerpo de su artículo, como una nota al final del texto, la autora presentaba una cuarta condición, afirmando que afrontará mejor el reto de la investigación colaborativa quien ya sepa cómo hacer una buena etnografía. No obstante, tiene que quedar bien claro que el trabajo colaborativo no debe entenderse como la "nueva manera correcta" de hacer etnografía (Field 2008: 42); simplemente es una lógica y una herramienta, o combinación de herramientas, que se adaptan mejor a cierto tipo de situaciones de investigación y que pueden responder mejor a determinadas preguntas, pero no es una receta. Ni siquiera hay una única forma de hacer etnografía colaborativa; hay etnografías colaborativas en plural, y en cada caso habrá que buscar las estrategias específicas que mejor se adapten al contexto particular. Lo fundamental es mantener en el centro de nuestras investigaciones el cuestionamiento sobre los modos de producción y validación del conocimiento: para qué y para quién se produce, cómo se produce, y cuáles son los criterios para considerar un determinado saber como legítimo (o como ilegítimo, no importante, desechable), y desplegar las estrategias metodológicas que nos permitan abrir otros horizontes para y desde nuestros proyectos.

\section{Conclusiones}

El objetivo de las propuestas colaborativas, apoyándose en ese desplazamiento central que supone pasar de una relación sujeto/objeto a una relación entre sujetos en proceso, es romper con las asimetrías propias de las situaciones de investigación, desbordar las lógicas disciplinarias auto-referenciales y lograr que nuestros proyectos sean útiles y relevantes para las personas con las que trabajamos. Insisto en que estas propuestas no van a ser aplicables a todas las situaciones de investigación, pero considero que en el caso concreto del estudio de la acción colectiva son sin duda una herramienta de gran valor.

Frente a la inercia auto-referencial de los estudios sobre movimientos sociales, pensar y producir conocimiento junto $y$ con los movimientos sociales demanda reconstruir espacios de diálogo y colaboración, y situar en el centro de la investigación los temas y las preguntas que emerjan de dicho diálogo. Es obvio que ambas dimensiones son distintas, academia y movimientos sociales no son lo mismo y no tienen porqué serlo, pero sin duda es posible articular procesos de escucha y conversaciones que permitan crear proyectos parcialmente compartidos. Estaríamos participando así en ese reto epistemológico y metodológico para la antropología que, como decía Escobar más arriba, remite a la dimensión política de la escritura etnográfica: para quién y cómo escribimos.

Por otro lado, quiero reiterar que situar la relevancia en el centro de nuestros proyectos, intentar que sean útiles para la gente con la que estamos trabajando, y hacer una etnografía de calidad, que pueda aportar respuestas y -sobre todo- preguntas inteligentes, no son incompatibles. No hay que elegir entre estas dos opciones. Todo lo contrario; pensar junto y con las personas que están dando cuerpo a esos nuevos protagonismos sociales es la mejor manera posible de alcanzar una comprensión más rica de estos fenómenos. Si nuestras propuestas están integradas dentro de las dinámicas internas de los movimientos, 
eso nos permitirá participar en situaciones de discusión y análisis colectivo que multiplicarán la riqueza y la complejidad de nuestras investigaciones. Tomarse en serio el carácter de compañeros epistémicos de las personas con las que trabajamos implica poner en el centro los momentos en los que ellos y ellas reflexionan colectivamente sobre sus propias categorías de sentido, y en los que las redefinen y resignifican. En unas redes y experiencias donde la reflexión conjunta sobre la práctica política es entendida en sí misma como una práctica política, estos encuentros son un contexto de máximo interés para comprender la reinvención de la acción colectiva, ya que es ahí donde se reactivan los procesos de experimentación en torno a las formas de hacer. Ése es el laboratorio donde se tejen las lógicas y los dispositivos emergentes: conversaciones, relaciones, conflictos y decisiones en las que se elaboran y se ponen en circulación categorías -formas de percibir y nombrar el mundo- que intentan catalizar la movilización y que son centrales para entender cómo ésta toma forma, se hace visible y va cambiando. Un proceso siempre en transformación mediante el que los movimientos construyen sentido. Ahí es donde tenemos que situarnos escuchando, acompañando y aprendiendo, en esa reflexividad creativa y compartida, en esa lógica de la artesanía de la política y de la investigación. Es ahí también donde seremos capaces de plantear preguntas con las que no solo la academia, sino también los y las protagonistas de estas experiencias, agentes reflexivos en permanente redefinición, quieran seguir caminando.

Hemos visto como el modelo dominante en el estudio de la acción colectiva ha ido perdiendo esta conexión, y al hacerlo ha profundizado las asimetrías que he tratado de problematizar en estas páginas: sujeto/objeto, teoría/práctica, saberes expertos/saberes cotidianos, etc. Hemos destacado también lo que la antropología puede aportar a este campo de análisis, y sabemos además que está empezando a hacerlo con mayor intensidad; como he mencionado proliferan los espacios de encuentro y la producción de textos en este sentido, y justamente por eso estamos en el mejor momento para replantear la discusión sobre las lógicas y prácticas colaborativas de investigación. Perder cierto grado de control sobre los proyectos en los que nos implicamos no solo no es un problema, sino que nos sitúa ante una oportunidad extraordinaria: imaginemos una etnografía sobre las formas emergentes de acción colectiva que resultara desbordada -llevada más allá de sus propios límites epistemológicos y metodológicos- por esas mismas prácticas emergentes de acción colectiva, ¿qué más podríamos pedir como investigadores e investigadoras?

\section{Notas}

1. Me refiero obviamente a estas formas emergentes de la acción colectiva; para otros actores y otro tipo de reivindicaciones las identidades fuertes siguen siendo fundamentales. Por poner un ejemplo cercano, tan solo hay que pensar en los diferentes proyectos de carácter nacionalista que están en disputa en el estado español, para los que la cuestión identitaria es absolutamente clave. Esto no invalida mis argumentos, sino que remite a la imposibilidad de explicar la heterogeneidad de los movimientos sociales desde una perspectiva o teoría unitaria (Santos 2001: 180).

2. Ver el trabajo de Fernández-Savater en http://blogs.publico.es/fueradelugar/tag/apuntes-de-acampadasol y http://www.eldiario.es/interferencias/ficcion-politica-15-M 6 71452864.html. Así como los materiales producidos por el programa Sociedad Civil y Comunicación de la UOC http://civilsc.net; y el grupo de investigación DatAnalysis15M http://datanalysis15m.wordpress.com.

3. Es el caso de V de Vivienda, o de la red de Oficinas de Derechos Sociales. Sin embargo, y por señalar antecedentes en un sentido más amplio, creo que es importante destacar el papel jugado por el despliegue del movimiento global, que marcó un punto de no retorno en relación a la primacía de las formas de organización en red, horizontales, flexibles, descentralizadas y sin una estructura ni un sujeto político (ni una ideología ni una identidad cerradas) que pudieran sobre-codificar los procesos de coordinación autónoma de las iniciativas que daban vida a ese heterogéneo movimiento de movimientos (Wainwright et al. 2007). Por otro lado, junto a las semejanzas hay también diferencias notables; el movimiento global tenía un carácter eminentemente nómada, los y las militantes se desplazaban de un lugar a otro del planeta señalando los centros políticos, económicos y militares de la gobernanza transnacional -las cumbres del G8, el Banco Mundial, el FMI, la OTAN, la OMC, la UE, etc.- o para asistir a los encuentros del propio movimiento: el Foro Social Mundial o los múltiples foros regionales. Por el contrario, en el ciclo actual las 
protestas están claramente territorializadas, y retoman además como objetivo prioritario de intervención las instituciones y los actores estatales y locales; esto no quiere decir que no haya referencias a otros niveles, como vemos en los países del sur de Europa en relación a las medidas adoptadas por el BCE, la Comisión Europea y el FMI, pero cada una de las luchas -la llamada Primavera Árabe, o las protestas de distinta intensidad en Islandia, Portugal, Grecia, México, Senegal, Turquía, Reino Unido, Israel, España, Estados Unidos o Brasil- respondían a la especificidad de sus propias coordenadas estatales (eso no impide, por otro lado, que se reconozcan a sí mismas como parte de una ola global más amplia, en cuyo interior las influencias son obvias y circulan -a través principalmente de las redes sociales- con gran rapidez de unas experiencias a otras).

4. Consultar el manifiesto completo de Democracia Real Ya! en http://www.democraciarealya.es/manifiestocomun

5. En inglés en el original, traducción propia.

6. En inglés en el original, traducción propia.

7. En inglés en el original, traducción propia.

8. Las principales revistas en lengua inglesa en el campo de estudio de los movimientos sociales siguen esta pauta, en especial Mobilization, fundada en 1996, y considerada como la más importante en este ámbito. La excepción a la regla es la revista Interface: a journal for and about social movements, activa desde 2009 y que nace con el objetivo explícito de romper con esa auto-referencialidad disciplinaria; ver http://www.interfacejournal.net/who-we-are/

9. Esto no debería entenderse como una defensa de las fronteras disciplinares; considero que toda investigación en ciencias sociales debe ser transdisciplinar. Es únicamente un llamamiento a desestabilizar las posiciones hegemónicas en el estudio de la acción colectiva, y un intento de mostrar lo que la antropología podría aportar en ese proceso.

10. En inglés en el original, traducción propia.

11. Más recientemente, se constituyó el grupo temático Antropología y Movimientos Sociales, dentro del Instituto Madrileño de Antropología, que en marzo de 2013 organizó el seminario "Entre la participación y la reflexividad: antropología y movimientos sociales", con contenidos similares a los que intento poner en discusión en este artículo. Ver http://antropologiaymovimientossociales.wordpress.com/2013/03/03/entre-laparticipacion-y-la-reflexividad-antropologia-y-movimientos-sociales/

\section{Ver http://www.ujaen.es/huesped/rae/}

13. Ver http://culanth.org/fieldsights/63-occupy-anthropology-and-the-2011-global-uprisings

\section{Ver http://www.prototyping.es/tag/15m.}

\section{Ver http://muse.jhu.edu/journals/collaborative_anthropologies/toc/cla.1.html}

16. http://bjsonline.org/2011/12/understanding-the-occupy-movement-perspectives-from-the-social-sciences/

17. Estas reflexiones surgen a partir del trabajo de campo realizado durante mi tesis doctoral, actualmente en fase de escritura, y que ha consistido en una aproximación etnográfica a la red de Oficinas de Derechos Sociales (ODSs), creada en la segunda mitad de la década del 2000, pero que tenía su origen al interior de redes y comunidades de activismo que compartían una amplia trayectoria previa de proyectos comunes en torno a la precariedad, a la cuestión de las migraciones y el régimen de fronteras, y a la experimentación en torno a las formas de hacer política desde los movimientos sociales. A nivel metodológico, el trabajo de campo de la investigación se desarrolló en 10 nodos de la red situados en Málaga, Zaragoza, Terrassa, Sevilla, Pamplona/Iruña, Barcelona y Madrid; y se basó en: los materiales elaborados por los y las integrantes de la red (informes internos, correos electrónicos, textos de reflexión, etc.); episodios de observación participante en múltiples contextos; una primera fase de entrevistas en profundidad realizadas en los distintos nodos; y por último, y aquí es donde tomaba forma la dimensión colaborativa, en una segunda fase de talleres de discusión y análisis colectivo, diseñados y elaborados (tanto a nivel 
metodológico como de contenidos) junto y con los y las activistas de la red a partir de los materiales producidos en la primera fase de trabajo de campo. Sobre investigaciones desarrolladas desde la etnografía colaborativa, ver también los trabajos de Leyva y Speed (2008), Vasco (2002), y el proyecto InterSaberes coordinado por Gunther Dietz en la Universidad Veracruzana, Mexico, http://www.intersaberes.org/

18. De hecho, el giro producido en el estudio de la acción colectiva hacia propuestas más dinámicas y relacionales ha hecho que un número creciente de autores y autoras reconozca y enfatice la importancia de la etnografía en el estudio de los movimientos sociales, debido a su capacidad para brindar acceso a los procesos políticos según se despliegan (McAdam 2003, McAdam, Tarrow y Tilly 2008), pero incluso cuando se comparte la herramienta el uso que se hace de la misma y la lógica desde la que se emplea son muy diferentes. Ni los objetivos, ni las preguntas ni las preocupaciones son coincidentes, y ésa es la razón por la que me parece importante alejarse de las orientaciones dominantes en el estudio de los movimientos sociales.

19. En inglés en el original, traducción propia.

20. En inglés en el original, traducción propia.

21. En inglés en el original, traducción propia.

22. Fueron los grupos estudiados por la antropología quienes, cansados ya de ser pensados y tratados como objetos, y de ver negada su capacidad y su legitimidad para construir sus propios relatos sobre sí mismos, empezaron a cuestionar de manera insistente el derecho (¿tú quién eres y por qué vienes a investigarme?) y las intenciones (¿para qué, para quién y cómo vas a hacerlo?) de los investigadores e investigadoras, exigiendo un mayor control tanto del proceso de construcción como del contenido de las representaciones que la antropología elaboraba en torno a sus formas de vida. Esta 'rebelión' de los sujetos subalternos/subalternizados, en la que tuvieron un papel fundamental las luchas anticoloniales y feministas, ya desde la década de 1960, ejerció un impacto incuestionable sobre la disciplina, problematizando y desestabilizando la autoridad -los discursos de verdad- de la antropología, y exigiendo la articulación de relaciones y prácticas más igualitarias, recíprocas y negociadas, diálogos compartidos (no exentos de conflictos, como toda relación social) frente a una antropología tradicionalmente extractiva y colonial. Este conjunto de críticas y debates se fue desarrollando tanto fuera como dentro de la academia, debido por un lado al empuje de esos movimientos, que lograban crear o conquistar espacios contrahegemónicos al interior de la universidad, y por otro al compromiso de científicos y científicas sociales que rechazaban la hegemonía de un canon que consideraban eurocéntrico, colonial y patriarcal. La combinación de estos factores impulsó la reflexión y experimentación en torno a metodologías que pudieran desbordar o revertir la división entre teoría y práctica, sujeto y objeto de investigación, etc.

23. En inglés en el original, traducción propia.

\section{Bibliografía}

Auyero, Javier

2005 “El oficio de la etnografía política (entrevista)", Iconos. Revista de Ciencias Sociales, n²2: 109-126.

Auyero, Javier (y Lauren Joseph)

2007 "Introduction: Politics under the Ethnographic Microscope", en Lauren Joseph (y otros) (eds.), Nen Perspectives in Political Ethnography. Nueva York, Springer: 1-13.

Bevington, Douglas (y Chris Dixon)

2005 "Movement-relevant Theory: Rethinking Social Movement Scholarship and Activism", Social Movement Studies, Volume 4, n 3: 185-208. 
Calle, Ángel

2013 La transición inaplazable. Salir de la crisis desde los nuevos sujetos políticos. Barcelona, Icaria.

Casas-Cortes, María Isabel (y otros)

2008 "Blurring Boundaries: Recognizing Knowledge-Practices in the Study of Social Movements", Anthropological Quarterly, nº 81: 1: 17-58.

Castells, Manuel

2012 Redes de indignación y esperanza. Los movimientos sociales en la era de Internet. Madrid, Alianza.

Chesters, Graeme (e lan Welsh)

2006 Complexity and Social Movements: Multitudes at the Edge of Chaos. Nueva York, Routledge.

Comaroff, John

2005 "Notes on Anthropological Method", Workshop on Interdisciplinary Standards for Systematic Qualitative Research.National Science Foundation, Washington, DC.

http://www.wjh.harvard.edu/nsfqual/papers.htm

Cox, Laurence (y Cristina Flesher-Fominaya)

2009 "Movement Knowledge: what do we know, how do we create knowledge and what do we do with it?", Interface a journal for and about social movements, Volume 1 (1): 1-20.

Croteau, David (y otros) (eds.)

2005 Rhyming Hope and History: Activists, Academics and Social Movement Scholarship. Minneapolis, University of Minnesota Press.

Cruells, Marta (y Pedro lbarra) (eds.)

2013 La Democracia del Futuro. Del 15M a la emergencia de una sociedad civil viva. Barcelona, Icaria.

Diani, Mario

2003 “Social Movements, Contentious Actions, and Social Networks: 'From Metaphor to Substance'?”, en Mario Diani y Doug McAdam (eds.), Social Movements and Networks: Relational Approaches to Collective Action. Oxford, Oxford University Press: 1-18.

Dietz, Gunther

2011 "Hacia una etnografía doblemente reflexiva: una propuesta desde la antropología de la interculturalidad", AIBR. Revista de Antropología Iberoamericana, nº 6 (1): 9-32.

Escobar, Arturo

1992 "Culture, Practice and Politics: Anthropology and the study of social movements", Critique of Anthropology, $\mathrm{n}^{\circ}$ 12: 395-432.

2008 Territories of Difference: Place, Movements, Life, Redes. Durham, Duke University Press.

Escobar, Arturo (y Michael Osterweil)

2009. "Movimientos sociales y la política de lo virtual. Estrategias deleuzianas", Tabula Rasa, n 10: 123161.

Fernández-Savater, Amador

2012 “El nacimiento de un nuevo poder social”, Hispanic Review, Volume 80, n 4: 667-681.

Field, Les W.

2008 "Side by Side or Facing One Another: Writing and Collaborative Ethnography in Comparative Perspective",Collaborative Anthropologies, Volume 1: 32-50.

Flacks, Richard

2004 "Knowledge for what? Thoughts on the state of social movement studies", en Jeff Goodwin y James M. Jasper (eds.), Rethinking Social Movements: Structure, Meaning, and Emotion. Lanham, Rowman \& Littlefield Publishers, Inc.: 135-153. 
Flesher, Cristina

2010 "Creating Cohesion from Diversity: The Challenge of Collective Identity Formation in the Global Justice Movement", Sociological Inquiry, Vol. 80, n³: 377-404.

Goodwin, Jeff (y Gabriel Hetland)

2009 "The Strange Disappearance of Capitalism from Social Movement Studies", Paper presented at the American Sociological Association Annual Meeting, Hilton San Francisco, San Francisco, CA.

http://www2.asanet.org/sectionchs/09conf/goodwin.pdf

Greenwood, Davydd

2000 "De la observación a la investigación-acción participativa: una visión crítica de las prácticas antropológicas", Revista de Antropología Social, n 9: 27-49.

Grimberg, Mabel (y otros)

2011 Etnografía de tramas políticas colectivas: Estudios en Argentina y Brasil. Buenos Aires, Antropofagia.

Hale, Charles R.

2008 "Introduction", en Charles R. Hale (ed.), Engaging contradictions: theory, politics, and methods of activist scholarship. Berkeley, University of California Press: 1-28.

Hammersley, Martyn (y Paul Atkinson)

1994 Etnografía. Métodos de investigación. Barcelona, Paidós.

Hannerz, Ulf

2010 Anthropology's World. Life in a Twenty-First-Century Discipline. London, Pluto Press.

Holmes, Brian

2007 "Continental Drift: Activist Research, From Geopolitics to Geopoetics", en Stevphen Shukaitis y David Graeber (eds.), Constituent Imagination: Militant Investigations, Collective Theorization. Oakland, AK Press: $39-43$.

Holmes, Douglas R. (yGeorge E. Marcus)

2008 "Collaboration Today and the Re-Imagination of the Classic Scene of Fieldwork Encounter", Collaborative Anthropologies, Volume 1: 81-101.

Juris, Jeffrey

2007 "Practicing Militant Ethnography with the Movement for Global Resistance in Barcelona", en Stevphen Shukaitis y David Graeber (eds.), Constituent Imagination: Militant Investigations, Collective Theorization. Oakland, AK Press: 164-176.

Juris, Jeffrey (y Alex Khasnabish) (eds.)

2013 Insurgent Encounters: Transnational Activism, Ethnography, and the Political. Durham, Duke University Press.

Lassiter, Luke Eric

2005 The Chicago Guide to Collaborative Ethnography. Chicago, University of Chicago Press.

Latour, Bruno

2005 Reassembling the Social. An Introduction to Actor-Network-Theory. Oxford, Oxford University Press.

Lazzarato, Maurizio

2006 Por una política menor. Acontecimiento y política en las sociedades de control. Madrid, Traficantes de Sueños.

Leyva, Xochitl (y Shannon Speed)

2008 "Hacia la investigación descolonizada: nuestra experiencia de co-labor", en Xochitl Leyva (y otras) (coords.), Gobernar (en) la diversidad: experiencias indígenas desde América Latina. Hacia la investigación de co-labor. México D.F., CIESAS-FLACSO: 34-59. 
McAdam, Doug

1994, "Cultura y movimientos sociales", en Enrique Laraña y Joseph Gusfield (orgs.), Los nuevos movimientos sociales: de la ideología a la identidad. Madrid, CIS: 43-67.

McAdam, Doug

2003 "Beyond structural analysis: towards a more dynamic understanding of social movements", en Mario Diani y Doug McAdam (eds.), Social Movements and Networks: Relational Approaches to Collective Action. Oxford, Oxford University Press: 281-298.

McAdam, Doug (y otros)

2008 “Methods for Measuring Mechanisms of Contention”, Qualitative Sociology, $\mathrm{n}^{\circ}$ 31: 307-331.

Mcdonald, Kevin

2002 "From Solidarity to Fluidarity: Social movements beyond 'collective identity' - the case of globalization conflicts", Social Movement Studies: Journal of Social, Cultural and Political Protest, 1:2: 109-128.

Melucci, Alberto

1989 Nomads of the Present. Social Movements and Individual Needs in Contemporary Society. Londres, Hutchinson.

Mendiola, Ignacio

2003 "Hacia una redefinición de los movimientos sociales: macro-actores proxémicos". Athenea Digital, $\mathrm{n}^{\circ}$ 4: 68-86.

Meyer, David S.

2005 "Scholarship that might matter", en David Croteau (y otros) (eds.), Rhyming Hope and History: Activists, Academics and Social Movement Scholarship. Minneapolis, University of Minnesota Press: 191205.

Nash, June (ed.)

2005 Social Movements: An Anthropological Reader. Londres, Blackwell.

Norris, Pippa

2002 Democratic Phoenix: reinventing political activism. Cambridge, Cambridge University Press.

Osterweil, Michal (y Graeme Chesters)

2007 "Global Uprisings: Towards a Politics of the Artisan", en Stevphen Shukaitis y David Graeber (eds.),Constituent Imagination: Militant Investigations, Collective Theorization. Oakland, AK Press: 253262.

Pelbart, Peter Pal

2009 Filosofía de la deserción. Nihilismo, locura y comunidad. Buenos Aires, Tinta Limón.

Rabinow, Paul

2007 Reflections on Fieldwork in Morocco. Berkeley, University of California Press.

Rappaport, Joanne

2007 "Más allá de la escritura: la epistemología de la etnografía en colaboración", Revista Colombiana de Antropología, Volumen 43: 197-229.

Rappaport, Joanne

2008 "Beyond Participant Observation: Collaborative Ethnography as Theoretical Innovation", Collaborative Anthropologies, Volume 1: 1-31.

Santos, Boaventura de Sousa

2001 "Los nuevos movimientos sociales", Observatorio Social de América Latina nº 5: 177-184.

Santucho, Mario A.

2012 "Reinterpretar el mundo entero (entrevista)", en Alberto Arribas (y otros) (eds.), Tentativas, contagios, 
desbordes. Territorios del pensamiento. Granada, Editorial Universidad de Granada: 107-132.

Schatz, Edward

2009 "Ethnographic Immersion and the Study of Politics", en Edward Schatz (ed.), Political Ethnography: What Immersion Contributes to the Study of Power. Chicago, University of Chicago Press: 1-22.

Taibo, Carlos

2011 Nada será como antes: sobre el movimiento 15-M. Madrid, Libros de la Catarata.

2012 Que no se apague la luz: un diario de campo del 15-M. Madrid, Libros de la Catarata.

Vasco Uribe, Luis Guillermo

2002 Entre selva y páramo: viviendo y pensando la lucha india. Bogotá, Instituto Colombiano de Antropología e Historia.

Villasante, Tomás R.

2006 Desbordes Creativos. Estilos y estrategias para la transformación social. Madrid, La Catarata.

VVAA

2004 Nociones comunes. Experiencias y ensayos entre investigación y militancia. Madrid, Traficantes de Sueños.

Wainwright, Hilary (y otros) (eds.)

2007 Networked Politics: Rethinking political organisation in an age of movements and networks. Amsterdam, Transnational Institute.

http://www.tni.org/archives/reports_newpol_networkedpolitics 